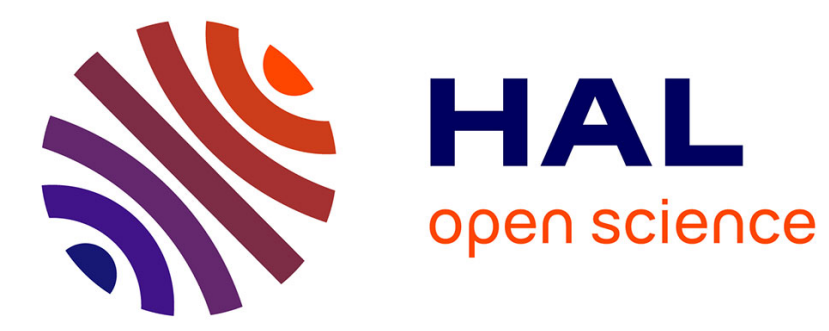

\title{
Transverse properties of dynamical systems
}

\author{
Jean Renault
}

\section{To cite this version:}

Jean Renault. Transverse properties of dynamical systems. Dynamical Systems and Asymptotic Combinatorics, 2004, St-Petersburg, Russia. hal-00083351

\section{HAL Id: hal-00083351 https://hal.science/hal-00083351}

Submitted on 30 Jun 2006

HAL is a multi-disciplinary open access archive for the deposit and dissemination of scientific research documents, whether they are published or not. The documents may come from teaching and research institutions in France or abroad, or from public or private research centers.
L'archive ouverte pluridisciplinaire HAL, est destinée au dépôt et à la diffusion de documents scientifiques de niveau recherche, publiés ou non, émanant des établissements d'enseignement et de recherche français ou étrangers, des laboratoires publics ou privés. 


\title{
Transverse Properties of Dynamical Systems
}

\author{
Jean Renault
}

\begin{abstract}
Roughly speaking, two dynamical systems are transversally equivalent if they have the same space of orbits; a property is transverse if it is preserved under transverse equivalence. Various notions of transverse equivalence have been defined: among them, similarity of measured groupoids, Morita equivalence of locally compact groupoids, stable orbit equivalence of measure equivalence relations. After reviewing some of these notions, we pass to discussing the Morita equivalence of groupoids and give examples and applications, in particular in connection with operator algebras.
\end{abstract}

\section{Introduction}

The dynamical systems of the present paper are pairs $(X, \mathcal{G})$ consisting of a space $X$ and a family $\mathcal{G}$, usually a group or a semi-group, of transformations of $X$. They also include foliations $(X, \mathcal{F})$. The orbits of a dynamical system (or the leaves of a foliation) form a partition of the space $X$. Roughly speaking, a transverse property of the dynamical system is a property of its orbit space $X / \mathcal{G}$ (or its leaf space $X / \mathcal{F}$ in the case of a foliation). The kind of question we may ask is, for example, "Are there transverse properties which distinguish two irrational rotations of the circle?" The answer is not obvious because their orbit spaces are singular and not amenable to classical analysis. Therefore, we need a precise definition saying when two orbit spaces are the same (one also says that the dynamical systems are "orbit equivalent"). Of course, it will depend on the way we view the orbit space, for example as a measured space or as a topological space.

Groupoids provide a convenient framework for giving such a definition. There are groupoids (usually one) naturally attached to the dynamical systems we consider here. After introducing a suitable notion of equivalence of groupoids, a transverse property of a dynamical system can be defined as a property of the equivalence class of its groupoid. This idea appears in the work of G. Mackey (cf. [Ma]) in the framework of ergodic theory (for a different purpose, namely the study of unitary group representations). In this context, he considers measured groupoids and the notion of equivalence he uses is similarity of measured groupoids; he defines

2000 Mathematics Subject Classification. Primary 37D35; Secondary 46L85.

Key words and phrases. Locally compact groupoids, $\mathrm{C}^{*}$-algebras, Morita equivalence, amenability, groupoid cohomology, Brauer group.

(C)2006 American Mathematical Society and International Press 
a virtual group as a similarity class of measured groupoids. This notion has been studied extensively by A. Ramsay (e.g. in $[\mathbf{R a}]$ ).

Specialized to countable standard measured equivalence relations, as defined by Feldman and Moore in $[\mathbf{F M}]$, similarity agrees with Kakutani equivalence. Here $X$ is a standard Borel space, $R$ is an equivalence relation on $X$ which has countable classes and a Borel graph (also denoted by $R$ ), and $[\mu]$ is the class of a $\sigma$-finite measure on $X$ quasi-invariant under $R$. The action of a countable group of Borel automorphisms of $X$ preserving the measure class $[\mu]$ provides such an equivalence relation (in fact, they can all be obtained that way). First, two countable standard measured equivalence relations $\left(X_{i}, R_{i},\left[\mu_{i}\right]\right), i=1,2$ are said to be isomorphic if the measured groupoids $\left(R_{i},\left[\mu_{i}\right]\right)$ are isomorphic; this amounts to saying that there exists a Borel isomorphism $\varphi: X_{1}^{\prime} \rightarrow X_{2}^{\prime}$, where $X_{i}^{\prime}$ is a conull set in $X_{i}$, sending $\mu_{1}$ onto a measure equivalent to $\mu_{2}$ and such that for all $x \in X_{1}^{\prime}, \varphi\left(R_{1}(x)\right)=R_{2}(\varphi(x))$. Two countable standard measured equivalence relations $\left(X_{i}, R_{i},\left[\mu_{i}\right]\right), i=1,2$ are said to be similar if there exists a countable standard measured equivalence relations $(Y, S,[\mu])$ and Borel subsets $Y_{i}, i=1,2$ of $Y$ having positive measure and meeting almost every orbit of $S$ such that $\left(X_{i}, R_{i},\left[\mu_{i}\right]\right)$ are respectively isomorphic to $\left(Y_{i}, S_{\mid Y_{i}},\left[\mu \mid Y_{i}\right]\right)$. The most studied case is when there is a probability measure $\mu$ on $X$ invariant under the equivalence relation $R$ (when the relation arises from the action of a countable group, this agrees with the usual definition of an invariant measure). One says then that $(X, R, \mu)$ is a probability measure preserving (p.m.p.) equivalence relation. An isomorphism of two ergodic p.m.p. equivalence relations $\left(X_{i}, R_{i}, \mu_{i}\right), i=1,2$ automatically preserves the invariant probability measures. In the non-ergodic case, one may want to add this condition. This notion of isomorphism of p.m.p. equivalence relations is crucial in the classification of p.m.p. actions of countable groups (in this context, one often says that the actions are orbit equivalent when their orbit equivalence relations are isomorphic), starting with the earlier work by Murray and von Neumann $[\mathbf{M N}]$ and followed by H. Dye [D1, D2], A. Vershik, R. Belinskaya and others. The recent survey $[\mathbf{G}]$ by D. Gaboriau gives a clear account of the present theory of orbit equivalence of group actions.

Let us consider now topological dynamical systems. We have in mind for example a locally compact group acting continuously on a locally compact space. Then there is a topological groupoid, called its semi-direct product, attached to such a dynamical system. There is a convenient notion of equivalence of topological groupoids, introduced in [Re2], which replaces Mackey's similarity of measured groupoids. Since it is prompted by the notion of Morita equivalence of $\mathrm{C}^{*}$-algebras introduced by M. Rieffel in [Ri] and gives an equivalence of categories, it is often called Morita equivalence of groupoids. It is the object of this communication.

The theory of foliations and the study of orbifolds teach us that, due to the possible presence of holonomy, it is more natural to introduce a groupoid rather than an equivalence relation. I should point out that, even when applied to a free action of a group, where the semi-direct product groupoid is an equivalence relation, the notions of isomorphism or equivalence of groupoids discussed in this talk are not the notions of topological orbit equivalence and Kakutani equivalence used by Giordano, Putnam and Skau in [GPS]. Indeed, let $\left(X_{i}, R_{i}\right), i=1,2$ be equivalence relations on topological spaces. Orbit equivalence in the sense of [GPS] is given by a homeomorphism $\varphi: X_{1} \rightarrow X_{2}$ such that for all $x \in X_{1}, \varphi\left(R_{1}(x)\right)=R_{2}(\varphi(x))$. In the context discussed here, the equivalence relations $R_{i}$ are topological groupoids 
and isomorphism requires that the induced bijection $\varphi \times \varphi: R_{1} \rightarrow R_{2}$ respects the topology, which is a stronger requirement. It should also be said that a conjugation of two dynamical systems gives an isomorphism of the corresponding groupoids but not conversely. On the other hand, the notion of equivalence of groupoids used here and its derived notion of transverse property agree with that used in the theory of foliations (see [H2] and [Ml, Section 2.7]).

The next section recalls the definition of Morita equivalence of $\mathrm{C}^{*}$-algebras and topological groupoids. One of the most basic transverse invariants is K-theory, which is briefly discussed in Section 3. Topological amenability, which is a transverse property, and its applications are reviewed in Section 4. The final Section 5 briefly discusses cohomology of topological groupoids and gives a construction of the Dixmier-Douady class of an elementary equivariant $\mathrm{C}^{*}$-bundle. This is interesting for our purpose since it makes good use of both Morita equivalence of $\mathrm{C}^{*}$-algebras and equivalence of groupoids. The definitions and results of Sections 2-4 are well known. Section 5 relies on [T2, TXL] and[KMRW]. Although I have no explicit reference for it, the construction of the Dixmier-Douady class presented here will look familiar to the specialists. It is based on a talk which I gave at a conference on groupoids and stacks held in Luminy, June 28-July 2nd 2004. I thank the participants of both conferences for stimulating discussions, and in particular J.-L. Tu and X. Tang.

\section{Morita equivalence of $\mathrm{C}^{*}$-algebras and groupoids}

2.1. $\mathbf{C}^{*}$-algebras. Let us recall the now classical notion of Morita equivalence of $\mathrm{C}^{*}$-algebras, introduced by $\mathrm{M}$. Rieffel in $[\mathbf{R i}]$. It is relevant here for several reasons: it will appear a few times and it has prompted the definition of equivalence of groupoids given below.

Definition 2.1 ([Ri, Definition 2.1]). Let $B$ be a $\mathrm{C}^{*}$-algebra. A right $B$ - $C^{*}$ module is a complex linear space $E$ endowed with

(i) a structure of right- $B$-module

$$
(x, b) \in E \times B \mapsto x b \in E
$$

(ii) a $B$-valued "inner product"

$$
(x, y) \in E \times E \mapsto<x, y>\in B
$$

satisfying

(a) it is $B$-linear in the second variable $y$,

(b) $\left\langle y, x>^{*}=<x, y>\right.$

(c) $\langle x, x\rangle$ is a positive element of $B$

(d) $\|x\|=\|<x, x>\|^{1 / 2}$ is a complete norm on $E$

We say that a $B$-C $C^{*}$-module $E$ is full if the linear span of the range of the inner product is dense in $B$.

One recognizes the definition of a Hilbert space when $B=\mathbf{C}$. The notions of bounded and compact linear operators can be defined in this new setting. Given two $\mathrm{C}^{*}$-modules $E$ and $F$, one defines $\mathcal{L}_{B}(E, F)$ as the space of bounded $B$-linear operators $T: E \rightarrow F$ which admit an adjoint, and $\mathcal{K}_{B}(E, F)$ as the norm-closure of the linear span of the rank-one operators $\theta_{x, y}$, where $x \in E, y \in E$ and for $z \in E$,

$$
\theta_{x, y}(z)=x<y, z>\text {. }
$$


For $F=E$, one gets the $\mathrm{C}^{*}$-algebra $\mathcal{L}_{B}(E)$ and its closed ideal $\mathcal{K}_{B}(E)$.

Definition 2.2 ([Ri, Definition 4.19]). Let $A$ and $B$ be $\mathrm{C}^{*}$-algebras. An $(A, B)-C^{*}$-correspondence (or a correspondence from $A$ to $B$ ) is a right $B$ - $C^{*}$ module $E$ together with a $*$-homomorphism $\pi: A \rightarrow \mathcal{L}_{B}(E)$.

We shall usually view an $(A, B)$ - $\mathrm{C}^{*}$-correspondence as an $(A, B)$-bimodule. A $*$-homomorphism $\pi: A \rightarrow B$ defines an $(A, B)$-C*-correspondence, by considering $E=B$ as a right $B$-C ${ }^{*}$-module (then $\mathcal{L}_{B}(E)=B$ ). It is useful to view $\mathrm{C}^{*}$-correspondences as generalized $*$-homomorphisms. There is a composition of correspondences extending composition of $*$-homomorphisms: given a $\mathrm{C}^{*}$ correspondence $E$ from $A$ to $B$ and a $\mathrm{C}^{*}$-correspondence $F$ from $B$ to $C$, one can construct the $\mathrm{C}^{*}$-correspondence $E \otimes_{B} F$ from $A$ to $C$. It is the $C$-C $\mathrm{C}^{*}$-module obtained by separation and completion of the ordinary tensor product $E \otimes F$ with respect to the inner product

$$
<x \otimes y, x^{\prime} \otimes y^{\prime}>=<<x, x^{\prime}>_{B}, y^{\prime}>_{C}, \quad x, x^{\prime} \in E, y, y^{\prime} \in F ;
$$

the left $A$ action is given by $a(x \otimes y)=a x \otimes y$ for $a \in A$. Associativity holds up to isomorphism. More precisely, given $\mathrm{C}^{*}$-algebras $A, B, C, D$ and $\mathrm{C}^{*}$-correspondences $E$ from $A$ to $B, F$ from $B$ to $C$ and $G$ from $C$ to $D$, there is a canonical isomorphism $\left(E \otimes_{B} F\right) \otimes_{C} G \rightarrow E \otimes_{B}\left(F \otimes_{C} G\right)$.

We say that an $(A, A)$-correspondence $E$ is an identity if it is isomorphic to the correspondence $A$ (defined by the identity map) and that an $(A, B)$-correspondence $E$ is invertible if there exists a $(B, A)$-correspondence $F$ such that $E \otimes_{B} F$ and $F \otimes_{A} F$ are identity correspondences. One has the following easy characterization of invertible correspondences.

Proposition 2.3 ([Ri, Section 6$])$. Let $A$ and $B$ be $C^{*}$-algebras and let $E$ be a linear space. Then the following conditions are equivalent:

(i) $E$ is invertible $(A, B)$ - $C^{*}$-correspondence,

(ii) $E$ is a full right $B$ - $C^{*}$-module and there is a $*$-isomorphism $\pi: A \rightarrow$ $\mathcal{K}_{B}(E)$

(iii) $E$ is a full right $B$ - $C^{*}$-module and a full left $A$ - $C^{*}$-module such that for $a$ in $A, x, y, z$ in $E$ and $b$ in $B$,

(a) $(a x) b=a(x b)$

(b) ${ }_{A}<x, y>z=x<y, z>_{B}$

(c) $<a x, a x>_{B} \leq\|a\|^{2}<x, x>_{B}$

(d) ${ }_{A}<x b, x b>\leq\|b\|_{A}^{2}<x, x>$

Definition 2.4 ([Ri, Definition 6.10]). An $(A, B)$-bimodule as in the above proposition is called an $(A, B)$-Morita equivalence. Two $\mathrm{C}^{*}$-algebras $A$ and $B$ are said to be Morita equivalent if there exists an $(A, B)$-Morita equivalence.

We leave to the reader to check that $A^{n}$ is a $\left(M_{n}(A), A\right)$-Morita equivalence (where $M_{n}(A)$ is the $\mathrm{C}^{*}$-algebra of $n \times n$ matrices over $A$ ). Another related basic example is the following: let $C$ be a $\mathrm{C}^{*}$-algebra and let $e, f$ be two projections in $C$ such that $C e C=C f C$. Then $e C f$ is a $(e C e, f C f)$-Morita equivalence. One should note the analogy with the definition of Kakutani equivalence recalled in the introduction. Let $E$ be an $(A, B)$-Morita equivalence. We define an inverse $(B, A)$-Morita equivalence $\tilde{E}$ by a bijection $x \in E \mapsto \tilde{x} \in \tilde{E}$ and the following operations: 
(i) $\tilde{x}+\tilde{y}=\widetilde{(x+y)} \quad \widetilde{(\lambda x)}=\bar{\lambda} \tilde{x} \quad$ for $\quad x, y \in X \quad$ and $\quad \lambda \in \mathbf{C}$,

(ii) $b^{*} \tilde{x} a^{*}=\widetilde{a x b}$ for $(a, x, b) \in A \times E \times B$,

(iii) $\left\langle\tilde{x}, \tilde{y}>_{A}={ }_{A}<x, y>\right.$ and ${ }_{B}<\tilde{x}, \tilde{y}>=<x, y>_{B}$.

Then $E \otimes_{B} \tilde{E}$ [resp. $\tilde{E} \otimes_{A} E$ ] is isomorphic to the identity correspondence $A$ [resp. $B]$ via the inner product.

2.2. Groupoids. Let us review some of the definitions. More information for this section can be found in $[\mathbf{R e} 1, \mathbf{R e} 2, \mathbf{M R W}]$. The most concise way to define a groupoid is to say that it is a small category with inverses. More concretely, we have a set of units $G^{(0)}$, with elements denoted by $x, y, \ldots$, a set of arrows $G$, with elements denoted by $\gamma, \gamma^{\prime}, \ldots$, the range and the source maps $r, s: G \rightarrow G^{(0)}$, the product map from $G^{(2)}=\left\{\left(\gamma, \gamma^{\prime}\right) \in G \times G: s(\gamma)=r\left(\gamma^{\prime}\right)\right\}$ to $G$ and the inverse map $\gamma \in G \mapsto \gamma^{-1} \in G$. We view $G^{(0)}$ as a subset of $G$ so that we have $r(\gamma)=\gamma \gamma^{-1}$ and $s(\gamma)=\gamma^{-1} \gamma$. Let us give a few examples, arising mostly from dynamical systems.

Groups. This is the case when $G^{(0)}$ has a single element.

Equivalence relations. Suppose that $R$ is an equivalence relation on the set $X$. Then, $G^{(0)}=X, G=\{(x, y) \in X \times X: x R y\}$ is the graph of the equivalence relation (from now on, we shall simply write $G=R$ ); the range and source maps are respectively the first and the second projections, the product is given by $(x, y)(y, z)=(x, z)$ and the inverse by $(x, y)^{-1}=(y, x)$. The identification of $G^{(0)}$ as a subset of $G$ is given by the diagonal map $x \mapsto(x, x)$.

Group actions. This is the classical case of a dynamical system. A group $\Gamma$ acts on a space $X$ (for convenience here on the right). We denote by $(x, t) \in X \times \Gamma \mapsto$ $x t \in X$ the action map. Then we define $G^{(0)}=X$ and the groupoid of the action, also called the semi-direct product,

$$
G=X \rtimes \Gamma=\{(x, t, y) \in X \times \Gamma \times X: x t=y\} .
$$

The range and source maps are respectively given by $r(x, t, y)=x$ and $s(x, t, y)=$ $y$. The product map is given by $(x, t, y)\left(y, t^{\prime}, z\right)=\left(x, t t^{\prime}, z\right)$ and the inverse by $(x, t, y)^{-1}=\left(y, t^{-1}, x\right)$. Note that $G$ is a subgroupoid of the product groupoid $\Gamma \times(X \times X)$ where $X \times X$ is the graph of the trivial equivalence relation. The image of the projection of $G$ into $X \times X$ is the graph of the orbit equivalence relation. The action is free if and only if this map is one-to-one.

Endomorphisms. Suppose that we have a map $T$ of $X$ into itself, not necessarily invertible. We define $G^{(0)}=X$ and the groupoid of the endomorphism

$$
G=\left\{(x, m-n, y) \in X \times \mathbf{Z} \times X: m, n \in \mathbf{N}, T^{m} x=T^{n} y\right\} .
$$

The maps and operations are the same as above. When $T$ is invertible, $G$ is the groupoid of the action of $\mathbf{Z}$ given by $T$. The following subgroupoid of $G$ is also relevant:

$$
R=\left\{(x, y) \in X \times \mathbf{Z} \times X: \exists m \in \mathbf{N}, T^{m} x=T^{m} y\right\} .
$$

These groupoids have been studied in particular by A. Vershik, who also introduced a generalization of them, with the notion of polymorphisms ([V2], see also $[\mathbf{A r R}])$.

Foliations. We introduce briefly this important example, which goes back to C. Ehresmann. Let $\mathcal{F}$ be a foliation, i.e., a partition into leaves, of the space $X$. A path $\gamma$ with endpoints $s(\gamma)$ and $r(\gamma)$ traced on a leaf defines a holonomy; we 
identify two paths defining the same holonomy and denote by $[\gamma]$ the holonomy classes $[\gamma]$. Then we define $G^{(0)}=X$ and the holonomy groupoid

$$
G=\{(x,[\gamma], y): x, y \in X, r(\gamma)=x, s(\gamma)=y\} .
$$

The maps and operations are the same as above, with $[\gamma]\left[\gamma^{\prime}\right]=\left[\gamma \gamma^{\prime}\right]$, where $\gamma \gamma^{\prime}$ is the concatenated path. One also considers transverse holonomy groupoids: they are reductions $G_{\mid T}$ to a full (i.e., meeting every leaf) transversal $T \subset X$.

Groupoid actions can be defined as functors. More concretely, a left $G$ space, where $G$ is a groupoid, consists of a space $Z$, a projection map $r: Z \rightarrow G^{(0)}$ (assumed to be surjective) and an action map from $G * Z$ to $Z$, where $G * Z$ is the set of composable pairs $(\gamma, z)$ with $s(\gamma)=r(z)$, satisfying the usual associativity rules; the image of $(\gamma, z)$ is denoted by $\gamma z$. A right $G$-space is defined similarly. One says that the action is free if the equality $\gamma z=z$ implies that $\gamma$ is the unit $r(z)$.

Two basic examples of actions of a groupoid $G$ are, on one hand, its action on its unit space $G^{(0)}$ given by $\gamma y=x$ where $x=r(\gamma)$ and $y=s(\gamma)$ and, on the other hand, its action on itself by left multiplication. This latter action is always free.

The definitions of a topological groupoid and of a continuous action are straightforward: with above notations, $G, G^{(0)}$ and $Z$ are topological spaces and all the maps we have introduced are continuous. There is a slight difficulty here because one may have to consider non-Hausdorff groupoids, for example the holonomy groupoid of the Reeb foliation. For the sake of simplicity, we assume that these topological spaces are Hausdorff. There is a further assumption that we make: the range, source and projection maps are assumed to be open. We then say the topological $G$-space $Z$ is proper if the map from $G * Z$ (endowed with the topology induced by the product topology of $G \times Z)$ to $Z \times Z$ which sends $(\gamma, z)$ to $(\gamma z, z)$ is proper. For example the left action of $G$ on itself is always proper.

The examples given above provide topological groupoids. For example, the groupoid of a continuous group action, endowed with the topology induced by the product topology of $X \times \Gamma \times X$, is a topological groupoid. The holonomy groupoid of a smooth foliation $\mathcal{F}$ of dimension $p$ on a manifold of dimension $p+q$ has a natural structure of manifold of dimension $2 p+q$, which turns it into a topological groupoid. The groupoid of an endomorphism which is a local homeomorphism has also a natural topology (see for example $[\mathbf{A r R}]$ ).

Equivalence of topological groupoids can be presented in a fashion analogous to Morita equivalence of $\mathrm{C}^{*}$-algebras.

Definition 2.5. Let $G$ and $H$ be topological groupoids. A $(G, H)$-correspondence (also called a correspondence from $G$ to $H$ ) is a right free and proper $H$-space $Z$ endowed with a left action of $G$ such that its projection map $r: Z \rightarrow G^{(0)}$ gives a homeomorphism of $Z / H$ onto $G^{(0)}$.

A groupoid homomorphism $\phi: G \rightarrow H$ defines a $(G, H)$-correspondence

$$
Z=G^{(0)} * H=\left\{(x, \eta) \in G^{(0)} \times H: \phi^{(0)}(x)=r(\gamma)\right\},
$$

where $H$ acts on the right by $(x, \eta) \eta^{\prime}=\left(x, \eta \eta^{\prime}\right)$, and $G$ acts on the left by $\gamma(s(\gamma), \eta)=$ $(r(\gamma), \phi(\gamma) \eta)$. Correspondences appear under various names in the literature; they are called morphisms of quotient spaces in $[\mathbf{H S}]$, generalized morphisms in $[\mathbf{L 1}]$ and 
Hilsum-Skandalis maps in [Mr]. There is a composition of correspondences extending the composition of groupoid homomorphisms: given a $(G, H)$-correspondence $X$ and a $(H, K)$-correspondence $Y$, one can construct the $(G, K)$-correspondence $X \circ Y=X * Y / H$, where $X * Y$ is the subspace of $X \times Y$ consisting of pairs $(x, y)$ such that $s(x)=r(y)$ and $H$ acts by $(x, y) \eta=\left(x \eta, \eta^{-1} y\right)$. We say that a $(G, G)$ correspondence $Z$ is an identity if it is isomorphic to the correspondence $G$ (defined by the identity map) and that a $(G, H)$-correspondence $X$ is invertible if there exists a $(H, G)$-correspondence $Y$ such that $X \circ Y$ and $Y \circ X$ are identity correspondences. One has the following easy characterization of invertible correspondences.

Proposition 2.6. Let $G$ and $H$ be topological groupoids and let $Z$ be a topological space endowed with commuting left $G$ - and right $H$-actions. Then the following conditions are equivalent:

(i) $Z$ is an invertible $(G, H)$-correspondence.

(ii) The actions are free and proper; the projection map $r: Z \rightarrow G^{(0)}$ gives a homeomorphism of $Z / H$ onto $G^{(0)}$ and projection map $s: Z \rightarrow H^{(0)}$ gives a homeomorphism of $G \backslash Z$ onto $H^{(0)}$.

Definition 2.7 ([Re2, Section 3],[MRW, Definition 2.1]). A $(G, H)$-space as in the above proposition is called a $(G, H)$-equivalence. Two topological groupoids $G$ and $H$ are said to be equivalent if there exists a $(G, H)$-equivalence.

Let $Z$ be a free and proper right $H$-space. The graph $Z * Z$ of the equivalence relation defined by the projection map $s: Z \rightarrow H^{(0)}$ is a topological groupoid and so is its quotient $Z * Z / H$ by the diagonal action of $H$. This groupoid has an obvious left action on $Z$ which turns $Z$ into a $(Z * Z / H, H)$-equivalence. Conversely, if $Z$ is a $(G, H)$-equivalence, then $G$ is isomorphic to $Z * Z / H$.

ExAmPLE 2.8. Let $p: Z \rightarrow X$ be a surjective continuous and open map, where $Z, X$ are topological spaces. Then, $Z * Z$ (fibred product above $X$ ), with the topological groupoid structure inherited from $Z \times Z$ is equivalent to $X$ via $Z$. In particular, if $\mathcal{U}=\left\{U_{i}\right\}_{i \in I}$ is an open cover of $X$, if $Z=X_{\mathcal{U}}$ is the disjoint union of the $U_{i}^{\prime} s$ and if $p: Z \rightarrow X$ is the obvious map, we get the groupoid associated with the open cover:

$$
G_{\mathcal{U}}=\left\{(i, x, j): x \in U_{i j}\right\}
$$

which is equivalent to $X$.

ExAmPLE 2.9. Let $H$ be a closed subgroup of a topological group $G$. Then, the right action of $H$ on $G$ by right multiplication is free and proper. Moreover, the semi-direct groupoid $G \ltimes(G / H)$ acts on $G$ on the left (the projection map $r$ : $G \rightarrow G / H$ is the quotient map). This makes $G$ into a $(G \ltimes(G / H), H)$-equivalence. This equivalence between $H$ and $G \ltimes(G / H)$ is at the heart of the theory of induced representations.

ExAmple 2.10. More generally, let $P$ and $\Gamma$ be two closed subgroups of a topological group $G$. Then, the semi-direct product groupoids $\Gamma \ltimes(G / P)$ and $(\Gamma \backslash G) \rtimes P$ are equivalent via the equivalence $G$.

ExAMPLE 2.11. Let $T$ and $T^{\prime}$ be transversals of a foliation $(X, \mathcal{F})$ which meet each leaf. Then the holonomy groupoid $G=G(X, \mathcal{F})$ and its reductions to the transversals $G_{\mid T}$ and $G_{\mid T}$, are all equivalent. For example $G_{T^{\prime}}^{T}=r^{-1}(T) \cap s^{-1}\left(T^{\prime}\right)$ is a $\left(G_{\mid T}, G_{\mid T^{\prime}}\right)$ equivalence. Let us give as an elementary example the Kronecker 
foliation of irrational slope $\alpha$ on the 2 -torus $\mathbf{T}^{2}$. The reduction of its holonomy groupoid to a closed transversal given by a line of rational slope is the groupoid of an irrational rotation of angle $2 \pi \beta$, where $\beta$ is in the orbit of $\alpha$ under the action of $P S L(2, \mathbf{Z})$; moreover, any $\beta$ in this orbit can be obtained. Therefore, two irrational rotations of respective angles $2 \pi \beta$ and $2 \pi \beta^{\prime}$, where $\beta$ and $\beta^{\prime}$ are in the same orbit under $\operatorname{PSL}(2, \mathbf{Z})$, have equivalent groupoids.

The classifying space of a topological groupoid $G$, defined as the quotient $B G=$ $E G / G$ of a universal $G$-space $E G$ (say within the category of free and proper $G$ spaces) provides some of the most basic transverse invariants (see [H2, Section 3]). In the example of the irrational rotation, this classifying space is, up to homotopy equivalence, the 2 -torus itself. In general, it is not so accessible.

\section{3. $\mathrm{C}^{*}$-algebras and analytic K-theory}

As pointed out earlier, our point of view owes very much to the theory of operator algebras. The analogy of the presentation of Morita equivalence for $\mathrm{C}^{*}$-algebras and for topological groupoids can be given a mathematical form via a suitable form [Re1] of the Murray-von Neumann "group-measure construction" [MN]. We have to impose additional assumptions on our topological groupoids: we require that they are second countable and locally compact and that they have a Haar system.

Let us explain this notion. Suppose that $G$ is a locally compact groupoid and that $Z$ is a locally compact left $G$-space, with the projection map $r: Z \rightarrow G^{(0)}$. A $r$-system is a family $\left(\alpha^{x}\right), x \in G^{(0)}$, where $\alpha^{x}$ is a Radon measure on $Z^{x}=r^{-1}(x)$. It is said to be continuous if for all $f$ in the space $C_{c}(Z)$ of continuous complexvalued functions on $Z$ with compact support, the function $x \mapsto \alpha^{x}(f)$ is continuous. It is said to be invariant if for all $\gamma \in G$, the image of $\alpha^{s(\gamma)}$ by $\gamma: Z^{s(\gamma)} \rightarrow Z^{r(\gamma)}$ is $\alpha^{r(\gamma)}$. A Haar system for the locally compact groupoid $G$ is a continuous and invariant $r$-system $\left(\lambda^{x}\right), x \in G^{(0)}$ for the left $G$-space $G$. We implicitly assume that all $\lambda^{x}$ are non-zero.

Contrarily to the case of locally compact groups, neither existence nor uniqueness hold in general. Existence of a Haar system is a topological assumption on $G$. For example, if the range map $r: G \rightarrow G^{(0)}$ has countable fibers, $G$ has a Haar system if and only if $r$ is a local homeomorphism. Then, the counting measures form a Haar system and one says that $G$ is an étale groupoid. This is a large and interesting class of groupoids including groupoids of discrete group actions, groupoids of endomorphisms provided they are themselves local homeomorphisms and transverse holonomy groupoids. The groupoid $X \rtimes \Gamma$ of the continuous action of a locally compact groupoid $\Gamma$ on a locally compact space has a Haar system of the form $\lambda^{x}=\delta_{x} \times \lambda$, where $\lambda$ is a (left) Haar measure for $\Gamma$ and $\delta_{x}$ is the point mass at $x$. The holonomy groupoid of a foliation also has Haar systems, with measures in the Lebesgue class.

Suppose that $G$ is a locally compact groupoid with Haar system $\lambda$. Then $C_{c}(G)$ can be made into a $*$-algebra via the formulas:

$$
\begin{gathered}
f * g(\gamma)=\int f\left(\gamma \gamma^{\prime}\right) g\left(\gamma^{\prime-1}\right) d \lambda^{s(\gamma)}\left(\gamma^{\prime}\right), \\
f^{*}(\gamma)=\overline{f\left(\gamma^{-1}\right)} .
\end{gathered}
$$


Then one defines the full $\mathrm{C}^{*}$-algebra $C^{*}(G, \lambda)$ as its universal $\mathrm{C}^{*}$-completion. One also defines the reduced $\mathrm{C}^{*}$-algebra $C_{r e d}^{*}(G, \lambda)$ by introducing the regular representation (it acts no longer on a Hilbert space, as in the case of a group, but on the $C_{0}\left(G^{(0)}\right)$-C'-module $\left.L^{2}(G, \lambda)\right)$. When $G=X \rtimes \Gamma$ comes from a group action, $C^{*}(G, \lambda)$ and $C_{r e d}^{*}(G, \lambda)$ are respectively the classical crossed-products $C^{*}(X, \Gamma)$ and $C_{r e d}^{*}(X, \Gamma)$.

TheOREM 3.1 ([MRW, Theorem 2.8]). Let $(G, \lambda)$ and $\left(H, \lambda^{\prime}\right)$ be second countable locally compact groupoids with Haar systems. Then, for any $(G, H)$-equivalence $Z, C_{c}(Z)$ can naturally be completed into a $\left(C^{*}(G, \lambda), C^{*}\left(H, \lambda^{\prime}\right)\right)$-Morita equivalence.

The same result (with the same proof) holds with the reduced $\mathrm{C}^{*}$-algebras. More generally, if $Z$ is a free and proper right $H$-space which has a $s$-system $\alpha$ (here, $s: Z \rightarrow H^{(0)}$ denotes the projection map), one can complete $C_{c}(Z)$ into a right $C^{*}\left(H, \lambda^{\prime}\right)-\mathrm{C}^{*}$-module $C^{*}(Z, \alpha)$. If moreover, $Z$ is a $(G, H)$-correspondence, $C^{*}(Z, \alpha)$ is also a right $C^{*}(G, \lambda)$-module and this makes it into a correspondence from $C^{*}(G, \lambda)$ to $C^{*}\left(H, \lambda^{\prime}\right)$. With suitable choices of systems of measures, this construction is functorial, in the sense that it respects composition up to isomorphism.

Corollary 3.2. Let $G$ be a second countable locally compact groupoid with Haar system $\lambda$. Then for $i=0,1$ the analytic $K$-theory groups $K_{i}\left(C^{*}(G, \lambda)\right)$ and $K_{i}\left(C_{\text {red }}^{*}(G, \lambda)\right)$ are transverse invariants.

This results from the theorem and the fact that an $(A, B)$-Morita equivalence of $\mathrm{C}^{*}$-algebras gives an isomorphism of their K-theory groups. These groups are called the analytic K-theory groups of the groupoid $G$. An early landmark was the computation by M. Pimsner and D. Voiculescu of these groups for the irrational rotation. As abelian groups $\left(K_{0}=\mathbf{Z}^{2}, K_{1}=\mathbf{Z}^{2}\right)$, they do not distinguish two irrational rotations. However, $K_{0}$ has a natural order and this ordered group is also invariant under Morita equivalence, hence it is also a transverse invariant. For the irrational rotation of angle $2 \pi \alpha$, this ordered group is $\mathbf{Z}+\alpha \mathbf{Z}$, with the order inherited from $\mathbf{R}$. Our earlier remark shows that this ordered group is a complete transverse invariant. At this point, let us briefly mention the Baum-Connes or assembly map. These authors have constructed a natural group homomorphism

$$
\mu: K_{\text {top }}^{*}(G) \rightarrow K_{*}\left(C_{\text {red }}^{*}(G, \lambda)\right)
$$

where the left-hand side group is the so-called geometrical K-theory group; it involves the universal proper $G$-space (see $[\mathbf{C}]$ or $[\mathbf{T 1}]$ for a precise definition). This map is compatible with groupoid equivalence. It has been shown to be an isomorphism in many cases.

\section{Amenability}

This notion, which plays an important rôle in the harmonic analysis of locally compact groups, can also be defined for dynamical systems. Here we shall only consider a definition of amenability for locally compact groupoids (topological amenability) and refer the reader to $[\mathbf{A n R}]$ for further information and references. Amenability of group actions also appears in V. Kaimanovich's talk.

The nicest kind of amenability is the following:

DeFinition 4.1. We say that a locally compact groupoid $G$ is properly amenable if it admits a Haar system $\left(m^{x}\right), x \in G^{(0)}$ consisting of probability measures $m^{x}$. 
When $G$ already admits a Haar system, this condition is equivalent to the properness of $G$, in the sense that $G^{(0)}$ is a proper $G$-space. In the case of a group, this amounts to saying that the group is compact and, in the case of a group action, that the action is proper. We look for a weakening of this condition.

Definition 4.2. We say that a locally compact groupoid $G$ is topologically amenable if it admits a continuous approximate invariant mean, i.e., a net $\left(m_{i}=\right.$ $\left.\left\{m_{i}^{x}\right\}_{x \in G^{(0)}}\right)$ of continuous systems of probability measures $m_{i}^{x}$ on $G^{x}$ which is approximately invariant in the sense that the function $\gamma \mapsto\left\|\gamma m_{i}^{s(\gamma)}-m_{i}^{r(\gamma)}\right\|_{1}$ tends to zero uniformly on the compact subsets of $G$.

In the case of the semi-direct product groupoid $G=X \rtimes \Gamma$ of a continuous action of a locally compact group $\Gamma$ on a locally compact space $X$, we identify $G^{x}$ with $\Gamma$ and view $m_{i}^{x}$ as a probability measure on $\Gamma$; the approximate invariance condition becomes: $(x, t) \mapsto\left\|t m_{i}^{x t}-m_{i}^{x}\right\|_{1}$ tends to zero uniformly on the compact subsets of $X \times \Gamma$. Amenability of the action of $\Gamma$ on $X$ is a synonym for amenability of the groupoid $G=X \rtimes \Gamma$. Any action of an amenable group is amenable. Non-amenable groups admit interesting amenable actions. For example, boundary actions of hyperbolic groups are amenable. Note that these actions can not preserve any probability measure. An important property of topological amenability is its invariance under topological equivalence of groupoids.

Theorem 4.3 ([AnR, 2.2.17]). Topological amenability of locally compact groupoids is a transverse property.

When $G$ is an amenable locally compact groupoid with Haar system, its full and its reduced $\mathrm{C}^{*}$-algebras coincide $[\mathbf{A n R}, 6.1 .8]$ and this $\mathrm{C}^{*}$-algebra is nuclear [AnR, 6.2.14]. Moreover, the Baum-Connes map is an isomorphism ([T1]). As an application of this notion, one deduces that groups which admit an amenable action on a compact space (e.g. boundary actions) satisfy the Novikov conjecture $[\mathbf{H R}]$. Amenability for measured groupoids had been introduced earlier by R. Zimmer $[\mathbf{Z 1}$, Z2]; among other equivalent definitions, there is one analogous to Definition 4.2 [Re1, II.3.4]. It is invariant under similarity.

As well known, there are striking results concerning amenable measured equivalence relations. One of them is that, for countable ergodic standard measured equivalence relations, amenability is equivalent to hyperfiniteness $[\mathbf{C F W}]$. Another, mentioned in the introduction, is the classification, up to isomorphism, of amenable countable ergodic standard measured equivalence relations. The status of amenable locally compact groupoids, even when restricted to minimal equivalence relations is very different. Th. Giordano, I. Putnam and C. Skau have initiated a program of classification of minimal actions of $\mathbf{Z}^{d}$ (and more general amenable groups) on the Cantor space, completed for $d=1[$ GPS $]$ and with partial results for $d=2$ (C. Skau) which uses, as said before, a weaker notion of orbit equivalence.

In some sense, AF equivalence relations $[\mathbf{R e} 4]$ can be viewed as a topological version of hyperfinite equivalence relations. These are the relations which can be obtained as tail equivalence relations of a Bratteli diagram. W. Krieger has shown in $[\mathbf{K r}]$ that they are classified up to isomorphism by their dimension range, therefore, up to equivalence, by their dimension group, which is the ordered group $K_{0}$ which appeared in the previous section. Thus, its dimension group is a complete transverse invariant for an AF equivalence relations. The analogous statement for $\mathrm{AF}$ algebras had been known earlier. Another class of nuclear $\mathrm{C}^{*}$-algebras has a 
good classification theory, namely the simple purely infinite ones are classified by their KK-theory (Kirchberg-Phillips). The $\mathrm{C}^{*}$-algebras associated with boundary actions of discrete groups enter into this class [A1] and are classified up to Morita equivalence by their K-theory. However, up to my knowledge, the classification of boundary actions up to groupoid equivalence has not been completed. A step into that direction is the computation by C. Anantharaman-Delaroche in [A2] of these K-theory groups for the action of a Fuchsian group on $P^{1}(\mathbf{R})$, which shows that non-conjugate actions can give isomorphic K-theory groups.

\section{Cohomology and the Dixmier-Douady class}

Since topological groupoids generalize both topological spaces and topological groups, it is worthwhile to define a cohomology theory which unifies cohomology of spaces and group cohomology. Grothendieck's equivariant sheaf cohomology, originally defined for discrete group actions, has been extended to étale topological groupoids by A. Haefliger $[\mathbf{H 1}]$ and by A. Kumjian $[\mathbf{K u}]$. As shown recently by J.-L. Tu in [T2], sheaf and Čech cohomology can be defined for arbitrary topological groupoids as a particular case of topological simplicial spaces. This definition subsumes the previous definitions.

Given a topological groupoid $G$, let us define a $G$-bundle $\mathcal{A}$ as a topological $G$-space with projection map $r: \mathcal{A} \rightarrow G^{(0)}$ such that its fibres $\mathcal{A}^{x}=r^{-1}(x)$ have an additional structure which is compatible with the topology of $\mathcal{A}$ and the action of $G$. For instance, we can speak of a $G$-bundle of groups or a $G$-bundle of Banach spaces. When the projection map is a local homeomorphism, one speaks of a $G$ sheaf instead of a $G$-bundle and of a stalk instead of a fibre. Given a topological groupoid $G$ and a $G$-sheaf $\mathcal{A}$ of abelian groups, we denote by $H^{n}(G, \mathcal{A}), n \in \mathbf{N}$ these cohomology groups (we do not here distinguish, as Tu does, sheaf and Čech cohomology). These are transverse invariants:

Theorem 5.1 ([T2, Section 8]). Let $G$ be a topological groupoid and let $\mathcal{A}$ be a $G$-sheaf $\mathcal{A}$ of abelian groups. Then, the cohomology groups $H^{n}(G, \mathcal{A}), n \in \mathbf{N}$ are invariant under Morita equivalence of topological groupoids.

For $n=0$ and $n=1$, these groups have a simple geometrical description: $H^{0}(G, \mathcal{A})$ is the group of (global) continuous $G$-equivariant sections of the projection map. In order to define $H^{1}(G, \mathcal{A})$, let us introduce the notion of $(G, \mathcal{A})$-affine space, which is quite similar to the notion of $(G, \mathcal{A})$-correspondence given in Definition 2.5. The difference is that we require that the maps $r, s: Z \rightarrow G^{(0)}$ coincide and we replace the commutation of the actions $\gamma(z a)=(\gamma z) a$ by the equivariance condition $\gamma(z a)=(\gamma z)(\gamma a)$ for $(\gamma, z, a) \in G * Z * A$. Then $H^{1}(G, \mathcal{A})$ is the group of isomorphism classes of $(G, \mathcal{A})$-affine spaces.

Just like for groups, the cohomology group $H^{2}(G, \mathcal{A})$ is related to extensions of $G$. Here are some definitions but we refer the reader to $[\mathbf{K M R W , ~ T 2 ]}$ for a more precise account. Let $G$ be a topological groupoid and let $A$ be a $G$-bundle of abelian groups. An extension of a topological groupoid $G$ by $A$ is a topological groupoid $E$ such that $E^{(0)}=G^{(0)}$, together with groupoid homomorphisms $i: A \rightarrow E$ (where $A$ is viewed as a groupoid) and $p: E \rightarrow G$ such that $i^{(0)}$ and $p^{(0)}$ are the identical map, $i$ is injective, $p$ is surjective, for $\sigma \in E, p(\sigma)$ is a unit if and only if $\sigma$ is in the image of $A$ and $\sigma i(a) \sigma^{-1}=i(p(\sigma) . a)$, where $\gamma . a$ is the image of $(\gamma, a) \in G * A$ by the action map $G * A \rightarrow A$. 
In order to integrate equivalence of groupoids, we also consider the extensions of the equivalent groupoid $G_{Z}=Z * Z / G$ by the induced group bundle $A_{Z}=Z * A / G$, where $Z$ is a right free and proper $G$-space. We say that the extensions $E_{i}$ of $G_{Z_{i}}$, where $i=1,2$, are equivalent if there is an equivalence $Z$ of the groupoids $E_{i}$, $i=1,2$, such that the actions of $A_{Z_{i}}$ agree and $A \backslash Z$ is isomorphic to $Z_{1} * Z_{2} / G$ as $\left(G_{Z_{1}}, G_{Z_{2}}\right)$-equivalence . Equipped with the Baer sum of extensions, the equivalence classes of such extensions form a group $\operatorname{Ext}(G, A)$ isomorphic to $H^{2}(G, \mathcal{A})$ ([T2, Proposition 5.6]), where $\mathcal{A}$ is the sheaf of germs of continuous sections of $A$.

The case when $A$ is the constant bundle $S=G^{(0)} \times \mathbf{S}^{1}$, where $\mathbf{S}^{1}$ is the circle group and the action of $G$ is trivial has received a special attention. The corresponding extensions are called twists over $G[\mathbf{K u}]$. They appear in particular in the theory of projective unitary representations of topological groups and in the Dixmier-Douady classification of certain continuous fields of elementary $\mathrm{C}^{*}$ algebras ([DD], see also [B, Chapter 4]). A general framework is given in [KMRW].

We present it here briefly and under a slightly different form. This theory concerns $G$-bundles of elementary $\mathrm{C}^{*}$-algebras. The notion of Morita equivalence of $\mathrm{C}^{*}$-algebras which we have reviewed in Section 2 is easily adapted to $G$-bundles of $\mathrm{C}^{*}$-algebras (some authors, e.g. $[\mathbf{L 2}]$, use the related notion of $G$-C $\mathrm{C}^{*}$-algebra): given a topological space $X$ and two bundles of $\mathrm{C}^{*}$-algebras $A \rightarrow X$ and $B \rightarrow X$, an $X$-Morita equivalence is a bundle of Banach spaces $E \rightarrow X$ and a structure of $\left(A_{x}, B_{x}\right)$-Morita equivalence on the fibre $E_{x}$ for all $x \in X$ such that all the structure maps are continuous. If $X=G^{(0)}$ and $A, B$ are $G$-bundles of $\mathrm{C}^{*}$-algebras, we shall say that $E$ is a $G$-Morita equivalence if it is a $G$-bundle of Banach spaces and the action of $G$ preserves the structure maps. Details can be found in $[\mathbf{R e} 3]$. By definition, an elementary $\mathrm{C}^{*}$-algebra $A$ is a $\mathrm{C}^{*}$-algebra isomorphic to the $\mathrm{C}^{*}$ algebra $\mathcal{K}(H)$ of compact operators on some Hilbert space $H$. Equivalently, it is a $\mathrm{C}^{*}$-algebra which is Morita equivalent to the $\mathrm{C}^{*}$-algebra $\mathbf{C}$.

Definition 5.2. A bundle of $\mathrm{C}^{*}$-algebras $A \rightarrow X$ is elementary if it is $X$ Morita equivalent to the constant bundle $X \times \mathbf{C}$. Equivalently, there exists a bundle of Hilbert spaces $H \rightarrow X$ such that the bundles $A \rightarrow X$ and $\mathcal{K}(H) \rightarrow X$ are isomorphic. A bundle of $\mathrm{C}^{*}$-algebras $A \rightarrow X$ is locally elementary if each point $x \in X$ has a neighborhood $U$ such that the reduced bundle $A_{\mid U} \rightarrow V$ is elementary.

The classical Dixmier-Douady invariant classifies locally elementary $\mathrm{C}^{*}$-bundles over $X$ up to $X$-Morita equivalence. It is an element $\delta_{X}(A) \in H^{2}(X, \mathcal{S})$, where $\mathcal{S}$ is the sheaf of germs of continuous functions from $X$ to the circle, which can be described as follows. Let $A \rightarrow X$ be a locally elementary $\mathrm{C}^{*}$-bundle. By definition, there exist an open cover $\mathcal{U}=\left\{U_{i}\right\}_{i \in I}$ of $X$ and for all $i$ a $U_{i}$-Morita equivalence $H_{i}$ from $A_{i}=A_{\mid U_{i}}$ to $U_{i} \times \mathbf{C}$. By choosing if necessary a finer cover, there exists for all $i$ a section $\xi_{i}$ of $H_{i} \rightarrow U_{i}$ such that $\left\|\xi_{i}(x)\right\|=1$ for all $x \in U_{i}$. It defines a section $p_{i}$ of $A_{i} \rightarrow U_{i}$ such that for all $x \in U_{i}, p_{i}(x)={ }_{A_{i}}<\xi(x), \xi(x)>$ is a one-dimensional projection of $A(x)$. For all $(i, j)$ such that $U_{i j}=U_{i} \cap U_{j}$ is non empty and $x \in U_{i j}$, we define $L_{i j}(x)=p_{i}(x) A(x) p_{j}(x)$. Then $L_{i j}$ is a Hermitian line bundle over $U_{i j}$. Given $i, j, k$ such that $U_{i j k}=U_{i} \cap U_{j} \cap U_{k}$ is nonempty and $x \in U_{i j k}$, the product in $A(x)$ gives a product $L_{i j}(x) \otimes L_{j k}(x) \rightarrow L_{i k}(x)$. For $x \in U_{i j}$, the involution in $A(x)$ gives an involution $L_{i j}(x) \rightarrow L_{j i}(x)$. For $v \in L_{i j}(x)$, we have $v v^{*}=\|v\|^{2} p_{i}(x)$ and $v^{*} v=\|v\|^{2} p_{j}(x)$. We define

$$
E=\left\{(i, x, j, v): x \in U_{i j}, v \in L_{i j}(x),\|v\|=1\right\} .
$$


Endowed with the multiplication $(i, x, j, v)(j, x, k, w)=(i, x, k, v w)$ and the inverse map $(i, x, j, v)^{-1}=\left(j, x, i, v^{*}\right)$, it is an extension of the groupoid associated with the open cover

$$
G_{\mathcal{U}}=\left\{(i, x, j): x \in U_{i j}\right\}
$$

by the constant bundle $X \times \mathbf{S}^{1}$. Moreover, these groupoids are topological. One can check that the class of this twist $E$ in $\operatorname{Ext}\left(X, X \times \mathbf{S}^{1}\right)$ does not depend on our choices. Viewed as an element of $H^{2}(X, \mathcal{S})$, it is the Dixmier-Douady invariant $\delta_{X}(A)$. The main result of $[\mathbf{D D}]$ is that $\delta_{X}(A)$ classifies the $\mathrm{C}^{*}$-bundle $A \rightarrow X$ up to $X$-Morita equivalence.

Let us consider next the locally elementary $\mathrm{C}^{*}$-bundles over $X$ which are endowed with a $G$-action, where $G$ is a given topological groupoid with unit space $G^{(0)}=X$. Let $A \rightarrow X$ be such a bundle. There exist an open cover $\mathcal{U}=\left\{U_{i}\right\}_{i \in I}$ of $X$ and sections $p_{i}$ over $U_{i}$ consisting of one-dimensional projections as above. We now introduce the groupoid

$$
G_{\mathcal{U}}=\left\{(i, \gamma, j): \gamma \in G, r(\gamma) \in U_{i}, s(\gamma) \in U_{j}\right\}
$$

which is equivalent to $G$. For $(i, \gamma, j) \in G_{\mathcal{U}}$, we define the Hermitian line

$$
L(i, \gamma, j)=p_{i}(r(\gamma)) A(r(\gamma))\left(\gamma p_{j}(s(\gamma))\right) .
$$

We have a product $L(i, \gamma, j) \otimes L\left(j, \gamma^{\prime}, k\right) \rightarrow L\left(i, \gamma \gamma^{\prime}, k\right)$ given by $a \otimes b \mapsto a(\gamma b)$ and an involution $L(i, \gamma, j) \rightarrow L\left(j, \gamma^{-1}, i\right)$ given by $a \mapsto \gamma^{-1}\left(a^{*}\right)$, which make

$$
E=\left\{(i, \gamma, j, v):(i, \gamma, j) \in G_{\mathcal{U}}, v \in L(i, \gamma, j),\|v\|=1\right\}
$$

into a twist over $G_{\mathcal{U}}$ defining the Dixmier-Douady invariant $\delta_{G}(A) \in H^{2}(G, \mathcal{S})$.

TheOREM 5.3 ([KMRW, Theorem 10.1]). Let $G$ be a topological groupoid. Two locally elementary $G$-C*-bundles are $G$-Morita equivalent if and only if they have the same Dixmier-Douady invariant.

In fact, the result of $[\mathbf{K M R W}]$ is more precise. It is first shown there that the $G$-Morita equivalence classes of locally elementary $G$-C ${ }^{*}$-bundles, with tensor product of $\mathrm{C}^{*}$-bundles as composition law, form a group; by analogy with a classical notion in algebra, this group is called the Brauer group of $G$ and is denoted by $\operatorname{Br}(G)$. Then, it is shown that the above Dixmier-Douady map is an isomorphism from $\operatorname{Br}(G)$ onto $H^{2}(G, \mathcal{S})$. The notion of K-orientation of a $G$-real vector bundle used in $[\mathbf{H S}]$ to construct elements of Kasparov KK-groups is related to the Dixmier-Douady invariant: a $G$-Euclidean vector bundle of even dimension (the general case follows by standard constructions) is K-oriented if and only if the Dixmier-Douady invariant $\delta_{G}(A) \in H^{2}(G, \mathcal{S})$ of the associated complex Clifford algebra bundle $A$ vanishes. These cohomology groups have been mostly studied for étale groupoids and in particular in the context of foliations $[\mathbf{H 1}]$ and for groupoids of endomorphisms $[\mathbf{D K M}]$. As a corollary of Theorem 5.1, these results can be applied to those groupoids which are equivalent to étale groupoids. There are also partial results on $\operatorname{Br}(G)$ when $G$ is the semi-direct product $X \rtimes \Gamma$ of the action of a group $\Gamma$ on a space $X$ relating it to the group cohomology of $\Gamma$ and the Cech cohomology of $X$ (e.g. [EN]). 


\section{References}

[A1] C. Anantharaman-Delaroche, Purely infinite $C^{*}$-algebras arising from dynamical systems, Bull. Soc. Math. France 125 (1997), 199-225.

[A2] C. Anantharaman-Delaroche, $C^{*}$-algèbres de Cuntz-Krieger et groupes fuchsiens, Proceedings of the 16th OT Conference (Timisoara, 1996), the Theta Foundation, 1997.

[AnR] C. Anantharaman-Delaroche, J. Renault, Amenable groupoids, Monographie de l'Enseignement Mathématique no. 36, Genève, 2000.

[ArR] V. Arzumanian, J. Renault, Examples of pseudogroups and their $C^{*}$-algebras, Operator Algebras and Quantum Field Theory, S. Doplicher, R. Longo, J. E. Roberts and L. Zsido, editors, International Press, 1997, pp. 93-104.

[AV] V. Arzumanian, A. Vershik, Star-algebras associated with endomorphisms, Operator algebras and group representations, Proc. Int. Conf., vol. 1, Pitman, Boston, 1984, pp. $17-27$.

[B] J.-L. Brylinski, Loop spaces, Characteristic classes and Geometric Quantization, Progress in Mathematics 107, Birkhäuser, Boston, 1993.

[C] A. Connes, Noncommutative geometry, Academic Press, 1994.

$[\mathrm{CFW}]$ A. Connes, J. Feldman, and B. Weiss, An amenable equivalence relation is generated by a single transformation, Ergodic Theory and Dynamical Systems 1 (1981), 431-450.

[D1] H. Dye, On groups of measure preserving transformations. I, Amer. J. of Math. 81 (1959), 119-159.

[D2] H. Dye, On groups of measure preserving transformations. II, Amer. J. of Math. 85 (1963), 551-576.

[DD] J. Dixmier, A. Douady, Champs continus d'espaces Hilbertiens et $C^{*}$-algèbres, Bull. Soc. Math. France 91 (1963), 227-284.

[DKM] V. Deaconu, A. Kumjian, P. Muhly, Cohomology of topological graphs and CuntzPimsner algebras, J. Operator Theory 46 (2001), 251-264.

[E] G. Elliott, On the classification of inductive limits of sequences of semisimple finitedimensional algebras, J. Algebra 38 (1976), 29-44.

[EN] S. Echterhoff, R. Nest, The structure of the Brauer group and crossed products of $C_{0}(X)$-linear group actions on $C_{0}(X, K)$, Trans. Amer. Math. Soc. 353 (2001), 36853712 .

[FM] J. Feldman, C. Moore, Ergodic equivalence relations, cohomologies, von Neumann algebras, I and II, Trans. Amer. Math. Soc. 234 (1977), 289-359.

[G] D. Gaboriau, On orbit equivalence of measure preserving actions, Rigidity in dynamics and geometry (Cambridge, 2000), Springer, Berlin, 2002, pp. 167-186.

[GPS] T. Giordano, I. Putnam, C. Skau, Topological orbit equivalence and $C^{*}$-crossed products, J. Reine Angew. Math. 469 (1995), 51-111.

[H1] A. Haefliger, Differentiable cohomology, CIME Lectures, Varenna 1976, (1979), 19-70.

[H2] A. Haefliger, Groupoides d'holonomie et classifiants, Structure transverse des feuilletages, Toulouse 1982, Astérisque 116 (1984), 70-97.

[HR] N. Higson, J. Roe, Amenable group actions and the Novikov conjecture, J. Reine Angew. Math. 519 (2000), 143-153.

[HS] M. Hilsum, G. Skandalis, Morphismes K-orientés d'espaces de feuilles et fonctorialité en théorie de Kasparov, Ann. Sci. E.N.S. 20 (1987), 325-390.

[KMRW] A. Kumjian, P. Muhly, J. Renault, D. Williams, The Brauer group of a locally compact groupoid, Amer. J. of Math. 120 (1998), 901-954.

[Kr] W. Krieger, On a dimension for a class of homeomorphismgroups, Math. Ann. 252 (1980), 87-95.

$[\mathrm{Ku}] \quad$ A. Kumjian, On equivariant sheaf cohomology and elementary $C^{*}$-bundles, J. Operator Theory 20(1988), 207-240.

[L1] P.-Y. Le Gall, Théorie de Kasparov équivariante et groupoïdes I, K-Theory 16 (1999), 361-390.

[L2] P.-Y. Le Gall, Groupoid $C^{*}$-algebras and operator K-theory, Groupoids in Analysis, Geometry, and Physics, Boulder 1999, Contemporary Mathematics (A.M.S.), 282 (2001), 137-146.

[Ma] G. Mackey, Ergodic theory and virtual groups, Math. Ann. 166 (1966), 187-207.

[Mk] I. Moerdijk, Orbifolds as Groupoids, an Introduction, K-Theory 18(1999), 235-253. 
[Ml] P. Molino, Riemannian foliations, Progress in Mathematics 73, Birkhäuser, Boston, 1988.

[MN] F. Murray, J. von Neumann, On rings of operators, Ann. of Math. 37 (1936), 116-229.

[Mr] J. Mrcun, Functoriality of the bimodule associated to a Hilsum-Skandalis map, KTheory 18 (1999), 235-253.

[MRW] P. Muhly, J. Renault, D. Williams, Equivalence and isomorphism for groupoid $C^{*}$ algebras, J. Operator Theory 17 (1987), 3-22.

[Ra] A. Ramsay, Virtual groups and group actions, Adv. in Math. 6 (1971), 253-322.

[Re1] J. Renault, A groupoid approach to $C^{*}$-algebras, Lecture Notes in Mathematics, vol. 793, Springer-Verlag Berlin, Heidelberg, New York, 1980.

[Re2] J. Renault, $C^{*}$-algebras of groupoids and foliations, Operator algebras and applications, Kingston 1980, Proc. Sympos. Pure Math. vol. 38 (1982), part I, pp. 339-350.

[Re3] J. Renault, Représentations des produits croisés d'algèbres de groupoïdes, J. Operator Theory 18 (1987), 67-97.

[Re4] J. Renault, AF equivalence relations and their cocycles, Proceedings of the OAMP Conference (Constanta, 2001), the Theta Foundation.

[Ri] M. Rieffel, Induced representations of $C^{*}$-algebras, Adv. in Math. 13 (1974), 176-257.

[T1] J. -L. Tu, La conjecture de Baum-Connes pour les feuilletages moyennables, K-theory 17 (1999), 215-264.

[T2] J. -L. Tu, Groupoid cohomology and extensions, preprint, arXiv: math.0A/0404257.

[TXL] J. -L. Tu, Ping Xu, C. Laurent, Twisted K-theory of Differentiable Stacks, preprint, arXiv: math.0A/0306138.

[V1] A. Vershik, Non measurable partitions, trajectory theory, operator algebras, Soviet. Math. Dokl. 199 (1971).

[V2] A. Vershik, Many-valued measure-preserving mappings (polymorphisms)and Markovian operators, translated from Zapiski Nauchnnyk Seminarov Leningradskogo Otdeleniya Matematicheskogo Instituta im. V. A.Steklova AN SSSR, 72 (1977), 26-61.

[Z1] R.J. Zimmer, Amenable ergodic group actions and an application to Poisson boundaries of random walks, J. Funct. Anal. 27 (1978), 350-372.

[Z2] R.J. Zimmer, Hyperfinite factors and amenable ergodic actions, Invent. Math. 41 (1977), 23-31.

Département de Mathématiques, Université d'Orléans, 45067 Orléans, France

E-mail address: renault@labomath.univ-orleans.fr 